\title{
Neutron Stars in a Varying Speed of Light Theory
}

\author{
A. W. Whinnett* \\ Astronomy Centre, \\ University of Sussex, \\ Falmer, \\ Brighton, \\ BN1 9QJ, \\ United Kingdom
}

January 11, 2019

\begin{abstract}
We study neutron stars in a varying speed of light (VSL) theory of gravity in which the local speed of light depends upon the value of a scalar field $\phi$. We find that the masses and radii of the stars are strongly dependent on the strength of the coupling between $\phi$ and the matter field and that for certain choices of coupling parameters, the maximum neutron star mass can be arbitrarily small. We also discuss the phenomenon of cosmological evolution of VSL stars (analogous to the gravitational evolution in scalar-tensor theories) and we derive a relation showing how the fractional change in the energy of a star is related to the change in the cosmological value of the scalar field.
\end{abstract}

PACS numbers: 04.40.Dg, 04.50.+h

\section{Introduction}

Recently, there has been interest in the possibility that the speed of light $c$ might have been larger in the past. The primary theoretical reason for considering this possibility is that such a variation in $c$ could solve the horizon and flatness problems of big-bang cosmology, without needing to postulate the existence of an inflationary epoch in the early history of the Universe 11. This purely theoretical work was given added impetus more recently after the discovery by Webb et al. 2] that, according to observations of wavelength shifts in the absorption lines of distant quasars, the fine structure constant $\alpha:=e^{2} /(\hbar c)$ seems to have been smaller in the past. This is consistent with the assumption that $c$ was larger in the past and, although more recent evidence [3] has constrained $\alpha$ variations more strongly than the Webb data, the possibility of a time varying $\alpha$ remains.

One can assume that any changes in the value of $\alpha$ are due to variations in one or both of $\hbar$ and $e$. However, the physical consequences of allowing either of these to vary are different from those that arise from a varying $c$ theory. For example, Avelino \& Martins [4 have shown that the horizon and flatness problems can only be solved by assuming that $c$ is varying. If instead one assumes that either of the other two constants is responsible for the variation of $\alpha$, the horizon and flatness problems remain.

${ }^{*}$ Email: visitor2@pact.cpes.susx.ac.uk 
To analyse the consequences of allowing a physical "constant" to vary, one needs a self consistent theory which is capable of describing this variation and its effect on other physical processes. Since $\alpha$, and by implication $c$, may change significantly only over cosmological time and distance scales, it is necessary to develop a theory of gravity which embodies such behaviour. This causes immediate problems when trying to formulate a varying speed of light (VSL) theory since the constancy of $c$ is a fundamental assumption of relativistic physics. Several attempts have been made to reconcile such a variation with a relativistic gravitational theory, including a bi-metric theory developed by Clayton \& Moffat [5] and Magueijo's scalar field theory [6, 7]. It is the latter that we shall focus on here.

In Magueijo's theory, the timelike coordinate in a coordinate chart no longer represents time as measured by a physical process and is instead replaced by a fourth length coordinate $\zeta$. When described in terms of this new coordinate, the structure and properties of a manifold are the same as in any relativistic theory of gravity and all of the rules of differential geometry remain unchanged. In particular, the manifold has a metric, a Riemann curvature tensor, and so on, all of whose components can be expressed in a $\left(\zeta, x^{i}\right)$ coordinate basis, where the $x^{i}$ are spatial coordinates. Time as measured by physical processes is not in general usable as a coordinate on the manifold. However, once a solution is found, physically measured time intervals can then be determined using the relation $d t=c^{-1} d \zeta$, where $c$ is treated as a scalar field on the manifold. The fact that $t$ is no longer necessarily a timelike coordinate is apparent when one considers the possibility that in a solution, surfaces of constant $c$ are not necessarily spacelike, so that $\nabla_{a} c$ will not be timelike. The VSL theory is superficially similar to Brans Dicke (BD) theory except that the scalar field, instead of determining the strength of the gravitational field, governs the rate of physical processes and, by implication, the rate at which clocks record time.

In any particular VSL theory, one would naturally expect spatial as well as temporal variations in $c$ in a spacetime with an inhomogeneous matter field. This is indeed the case, and Magueijo has analysed vacuum, spherically symmetric solutions representing both the weak field produced far from a central source and VSL black holes [7]. In this paper, we analyse the structure of neutron stars in Magueijo's VSL theory. The purpose of this is twofold: first, to determine the degree to which a VSL neutron star differs from its counterpart in general relativity (GR) or BD theory and second, to serve as a starting point for the theoretical treatment of binary pulsars in a VSL theory, with the ultimate aim of using binary pulsar data to constrain the parameters of the theory.

The plan of the paper is as follows. In Section 2 we give the action for the theory and derive the field equations for spherically symmetric spacetimes. In Section 3 we discuss the vacuum, exterior part of the solutions. In Section 4 we give the equation of state (EOS) of the fluid interior and discuss the issue of particle conservation, while in Section [5 we find the expression which describes the total energy of the solutions. In Section 6 we present the results of numerical integrations of the equations of structure and in Section 17 we discuss the evolution of the mass of a star in a cosmological setting. Finally, in Section 8 we present a few concluding remarks.

\section{Action and Field Equations}

In Magueijo's VSL theory, the local speed of light $c$ is determined by the value of a scalar field $\phi$ such that $c=c_{\infty} e^{\phi}$, where $c_{\infty}$ is the speed of light measured far from all gravitating sources. 
In units in which $G=1$, the action is given by ${ }^{1}$

$$
I=\frac{1}{16 \pi} \int_{\mathcal{V}} d^{4} x \sqrt{-g}\left[e^{\epsilon \phi}\left(\mathcal{R}-\kappa g^{a b} \nabla_{a} \phi \nabla_{b} \phi\right)+16 \pi e^{\beta \phi} L_{m}\right]-\frac{1}{8 \pi} \int_{\partial \mathcal{V}} d^{3} x \sqrt{h} e^{\epsilon \phi} \Theta,
$$

where $\mathcal{V}$ is an arbitrary volume of integration, $\partial \mathcal{V}$ its closed boundary, and $h_{a b}$ and $\Theta$ the induced metric and extrinsic curvature on this boundary. The surface term guarantees that the variational derivative of $I$ vanishes on $\partial \mathcal{V}$ and is important when determining the mass of an asymptotically flat spacetime. The constants $\epsilon, \beta$ and $\kappa$ are arbitrary. In [6] the condition $\epsilon-\beta=4$ was imposed since the units of the matter and gravitational terms of the action (11) differ by a factor of $c^{4}$. We shall assume this condition holds here. In addition, we shall assume that the "minimum coupling" assumption of [6] holds so that $L_{m}$ is independent of $\phi$.

The generalised Einstein equations which lead from this action are

$$
G_{a b}=\left(\kappa+\epsilon^{2}\right) \nabla_{a} \phi \nabla_{b} \phi-\left(\frac{1}{2} \kappa+\epsilon^{2}\right) g_{a b} \nabla^{c} \phi \nabla_{c} \phi+\epsilon\left(\nabla_{a} \nabla_{b} \phi-g_{a b} \nabla^{c} \nabla_{c} \phi\right)+8 \pi e^{(\beta-\epsilon) \phi} T_{a b},
$$

where $T_{a b}$ is the matter energy-momentum tensor, while the scalar field satisfies the wave equation

$$
\nabla^{a} \nabla_{a} \phi=-\epsilon \nabla^{a} \phi \nabla_{a} \phi+\frac{8 \pi G e^{(\beta-\epsilon) \phi}}{2 \kappa+3 \epsilon^{2}}\left(\epsilon T-2 \beta L_{m}\right) .
$$

The local matter conservation law for the theory is

$$
\nabla_{a} T^{a b}=-\beta\left(T^{a b}-g^{a b} L_{m}\right) \nabla_{a} \phi
$$

In the vacuum case, where $L_{m}=0$ and $T_{a b}$ vanishes, the action and field equations are identical to BD theory with

$$
\omega=\frac{\kappa}{\epsilon^{2}}, \quad \Phi=e^{\epsilon \phi},
$$

where $\omega$ and $\Phi$ are the BD coupling parameter and scalar field.

We are interested here in asymptotically flat, static and spherically symmetric solutions to the VSL field equations. We use isotropic coordinates and write the line element as

$$
d s^{2}=-B d \zeta^{2}+A\left(d r^{2}+r^{2} d \theta^{2}+r^{2} \sin ^{2} \theta\right)
$$

where $A$ and $B$ are functions of $r$, and $\zeta$ is a timelike coordinate with the units of length. We assume that the matter is composed of a perfect fluid with pressure $p$, energy density $\rho$ and a fluid 4-velocity $U^{a}$, for which the energy-momentum tensor has the usual form

$$
T^{a b}=(\rho+p) U^{a} U^{b}+p g^{a b} .
$$

In the coordinates we are using, $U^{a}=\left(B^{-1 / 2}, 0,0,0\right)$ and is orthogonal to the hypersurfaces of constant $\zeta$.

To integrate the resulting field equations, it is convenient to introduce an auxiliary function

$$
Z:=\frac{1}{2 B} \frac{d B}{d r}+\frac{1}{2 A} \frac{d A}{d r}
$$

In terms of these variables, the field equations reduce to the following set of coupled ODEs:

$$
A^{\prime \prime}=\frac{3 A^{\prime 2}}{4 A}-\frac{2 A^{\prime}}{r}+\epsilon \phi^{\prime}\left(A Z-\frac{A^{\prime}}{2}\right)-\frac{A \kappa \phi^{2}}{2}+8 \pi A^{2} e^{(\epsilon-\beta) \phi}\left[\frac{\rho\left(\epsilon^{2}-\epsilon \beta\right)+3 p \epsilon^{2}}{2 \kappa+3 \epsilon^{2}}-\rho\right],
$$

\footnotetext{
${ }^{1}$ The constants $\epsilon$ and $\beta$ we use here correspond to the constants $a$ and $b$ used by Magueijo [6, 7]. We use the former notation to avoid confusion with tensor indices.
} 


$$
\begin{gathered}
Z^{\prime}=-\frac{3 Z}{r}-Z^{2}-\epsilon \phi^{\prime}\left(Z+\frac{1}{r}\right)+8 \pi A e^{(\epsilon-\beta) \phi}\left[2 p+\frac{\rho \epsilon(\epsilon-2 \beta)-3 p \epsilon^{2}}{2 \kappa+3 \epsilon^{2}}\right], \\
\phi^{\prime \prime}=-\phi^{\prime}\left(\epsilon \phi^{\prime}+Z+\frac{2}{r}\right)+8 \pi A e^{(\epsilon-\beta) \phi}\left[\frac{3 p \epsilon-\rho(\epsilon+2 \beta)}{2 \kappa+3 \epsilon^{2}}\right]
\end{gathered}
$$

and

$$
p^{\prime}=-(\rho+p)\left(Z-\frac{A^{\prime}}{2 A}+\beta \phi^{\prime}\right)
$$

where the prime denotes $\frac{d}{d r}$.

\section{Exterior Solution, Boundary Values and Scaling Relations}

For a given set of values of $\epsilon, \beta$ and $\kappa$, the solutions are parameterised by the central density $\rho_{0}$. As in GR, the surface of a stellar solution is defined to be where the pressure vanishes and at this point the solution is matched to a vacuum exterior. Since the vacuum field equations for this theory are identical to BD theory, the vacuum solutions are also identical and, in terms of the variables we are using here, are given by

$$
\begin{gathered}
A=A_{\infty}\left(1+\frac{\mu}{r}\right)^{4}\left(\frac{r-\mu}{r+\mu}\right)^{2(1-C / \lambda-1 / \lambda)}, \\
Z=\frac{2 \mu}{\mu^{2}-r^{2}}
\end{gathered}
$$

and

$$
\phi=\phi_{\infty}+\frac{C}{\epsilon \lambda} \log \left(\frac{r-\mu}{r+\mu}\right),
$$

where the subscript " $\infty$ " denotes the value of quantities at spacelike infinity. In these equations $\mu, C$ and $\lambda$ are constants and satisfy the constraint

$$
\lambda^{2}=(C+1)^{2}-C\left(1-\frac{\kappa C}{2 \epsilon^{2}}\right) .
$$

Equations (13) and (14) together give the more familiar metric component

$$
B=B_{\infty}\left(\frac{r-\mu}{r+\mu}\right)^{2 / \lambda}
$$

These equations hold only when $\epsilon \neq 0$.

We shall assume that the interior neutron star solution matches smoothly to the above vacuum solution at the boundary of the star, in the sense that $A, Z, \phi$ and the derivatives of these functions are continuous at the boundary. We can then find the values of the parameters $\mu, C$ and $\lambda$ as follows. Differentiating eqns (13) to (15), one can show that

$$
X:=Z+\epsilon \phi^{\prime}=\frac{2 \mu^{2}}{r^{3}-\mu^{2} r} .
$$

Rearranging this equation, we have

$$
\mu=\sqrt{\frac{X r^{3}}{4+X r}}
$$


which allows us to determine the value of $\mu$. From eqn (14), one can show that

$$
\lambda=\left(Z-\frac{A^{\prime}}{2 A}\right) \frac{2 \mu}{r^{2}-\mu^{2}},
$$

which allows us to calculate $\lambda$ once $\mu$ has been found. Finally, from eqn (15), we have

$$
C=\frac{\lambda\left(r^{2}-\mu^{2}\right) \epsilon \phi^{\prime}}{2 \mu}
$$

which allows us to calculate $C$.

The minimal VSL theory mentioned in [7] is the one with $\epsilon=0$ and its field equations are much simpler than in the more general theory. For this particular case, the above equations have to be modified. The functions $A, B$ and $Z$ are given by eqns (13), (14) and (17) with the constant $C=0$. The scalar field is now given by

$$
\phi=q \log \left(\frac{r^{2}-\mu^{2}}{r^{2}+\mu^{2}}\right),
$$

where $q$ is a new constant, related to $\lambda$ and $\kappa$ by

$$
q^{2} \kappa=2-\frac{2}{\lambda^{2}}
$$

The Schwarzschild limit discussed in [7] is the special case of these equations in which $\lambda=1$. Note that in all cases in which $\epsilon=0$, the $\phi$ field need not be homogeneous.

Magueijo [7] has shown that, by carrying out a weak field analysis and interpreting the results within the framework of the $\mathrm{PPN}$ formalism, the parameters $\kappa, \epsilon$ and $\beta$ are related by the equation

$$
\gamma=\frac{\epsilon^{2}+\kappa+\frac{1}{2} \epsilon \beta}{2 \epsilon^{2}+\kappa-\frac{1}{2} \epsilon \beta}
$$

where $\gamma$ is the PPN parameter which governs, amongst other things, the deflection of light rays near to a massive body such as the Sun. Its limiting value is currently [8]

$$
|\gamma-1| \leq 0.0003
$$

Solving this eqn (24) for $\kappa$ we have

$$
\kappa=\frac{\epsilon(\epsilon-\beta)}{\gamma-1}+\frac{1}{2} \epsilon \beta-2 \epsilon^{2}
$$

which shows that $\kappa$ decreases as $\epsilon \rightarrow 0$ for the case when $\epsilon-\beta=4$. For values of $\epsilon$ of the order unity, eqn (26) restricts $\kappa$ to have a large value similar to that of $\omega$ in BD theory. However, if we allow $\epsilon$ to have an arbitrarily small value, there is no minimum value to $\kappa$.

\section{Equation of State and Particle Conservation}

We assume that the perfect fluid matter of the star consists solely of neutrons, each with rest mass $m$ and local number density $n$. Denoting the internal energy per particle by $\Pi$, the matter Lagrangian is then given by

$$
L_{m}=-m c^{2} n(1+\Pi)=-\rho,
$$


the latter equality defining the relationship between $n$ and $\rho$. Under the minimum coupling assumption outlined in [6], the particle rest energy $m c^{2}$ is constant and hence $L_{m}$ is independent of $\phi$.

We shall consider two equations of state. The first is that of a a non-interacting gas of Fermions, which may be given in the parametric form [9]

$$
\begin{array}{r}
p=\frac{m^{4} c^{5}}{24 \pi^{2} \hbar^{3}}\left[\chi\left(2 \chi^{2}-3\right) \sqrt{\chi^{2}+1}+3 \log \left(\chi+\sqrt{\chi^{2}+1}\right)\right] \\
\rho=\frac{m^{4} c^{5}}{3 \pi^{2} \hbar^{3}} \chi^{3} \sqrt{\chi^{2}+1}-p .
\end{array}
$$

This means that, in practice, eqn (12) becomes a differential equation for $\chi$. Note that, under the minimum coupling assumption of [6], both $\hbar$ and the product $m c$ scale with $\phi$ in the same way, so that $m c / \hbar$ is independent of $\phi$ and hence so are numerical coefficients of eqns (28).

More realistic equations of state are generally given in tabulated form and require complex calculations which take into account particle phase transitions at various densities. However, polytropic equations of state fitted to the tabulated data do exist and our second EOS is of this type. The pressure and density are given in the parameterised form

$$
\begin{gathered}
p=K n_{0} m\left(\frac{n}{n_{0}}\right)^{\Gamma} \\
\rho=n m+\frac{K n_{0} m}{\Gamma-1}\left(\frac{n}{n_{0}}\right)^{\Gamma},
\end{gathered}
$$

where the constants are chosen to have the values $n_{0}=1.0 \times 10^{44} \mathrm{~m}^{-3}, K=0.0195$ and $\Gamma=2.34$. The equations are parameterised by the particle number density $n$. This is the EOS used by Damour and Esposito-Farese in their investigation of strong field scalar effects in neutron stars [10] and is based on tabulated data given in [11]. As before, we assume that the minimum coupling assumption holds so these parameters are independent of the local value of $c$.

The first of our equations of state is far from realistic. However, we are interested here primarily in the effect a VSL theory has on the properties of a neutron star and we expect the main features of the solutions to be independent of the choice of EOS. In addition, our choice makes the results we find here easier to compare with the GR stars studied by Oppenheimer \& Volkoff [12] and the BD solutions of Salmona [13]. The second EOS, being more realistic, will be used to place tentative constraints on the theory.

Despite the presence of the factor of $e^{\beta \phi}$ in the matter part of the action (10), the conserved particle number $N$ for a neutron star solution is the same as in a metric theory, namely

$$
N=\int_{0}^{r_{s}} 4 \pi r^{2} A^{3 / 2} n d r
$$

where $n$ is the local particle number density. In the second EOS outlined above, this is the same quantity which parameterises those equations while in the simpler, non-interacting Fermion EOS, $n$ and $\chi$ are related by

$$
n=\left(\frac{m^{3} c^{3}}{3 \pi^{2} \hbar^{3}}\right) \chi^{3} .
$$

In this relation, the bracketed combination of parameters is independent of $c$ due to the minimal coupling assumption. 
We note here that for a non-perfect fluid matter source, the conserved charge should in general include explicit reference to the fact that $L_{m}$ is not universally coupled. One can show that, for matter Lagrangians $L_{m}(\psi)$ dependent on one or more matter fields $\psi$ which are invariant under a field transformation $\psi \rightarrow \psi+\delta \psi$, the Nöether current associated with the symmetry is given by

$$
J^{a}=e^{\beta \phi} I^{a},
$$

where $I^{a}$ is the corresponding current in a metric theory in which $L_{m}$ is universally coupled to the metric. This means that one should include a factor of $e^{\beta \phi}$ in the expression for the conserved charge. Such a factor appearing in the Nöether current would indicate a violation of a local energy conservation law. One can show that this does not occur for a perfect fluid source as follows. Dropping the assumption that the solutions are static and contracting eqn (4) with $U_{b}$ we have

$$
U_{b} \nabla_{a} T^{a b}=-\beta U_{b}\left[(\rho+p) U^{a} U^{b}+(p+\rho) g^{a b}\right] \nabla_{a} \phi
$$

which vanishes. Hence, at least for a perfect fluid, the non-spatial components of eqn (4) are trivially zero and the right hand term contributes only to the pressure gradient. Thus there is no violation of the local energy conservation law in this case.

\section{Mass}

Since the solutions are spherically symmetric and asymptotically flat, one may write the ADM mass as the integral

$$
M_{A D M}=\int_{0}^{\infty} 4 \pi r^{2} G_{a b} U^{a} U^{b} d r .
$$

This quantity is independent of the theory of gravity being considered and its value is determined solely by the metric. For both BD and VSL theories, from eqn (13), the ADM mass is also given by

$$
M_{A D M}=\frac{2(C+1) \mu}{\lambda} .
$$

The Poisson mass, discussed in [7, may be defined by the relation

$$
M_{P}:=\lim _{r \rightarrow \infty}\left(\frac{1}{2} r^{2} B^{\prime}\right)=\frac{2 \mu}{\lambda},
$$

where the second equality follows from using eqn (17).

In theories of gravity other that GR, $M_{A D M}$ does not describe the total, physical energy of an asymptotically flat solution. For example, in BD theory the energy of a spherically symmetric solution is the Tensor mass [14]

$$
\widetilde{M}_{T}:=\frac{1}{\Phi_{\infty}}\left[M_{A D M}-\frac{1}{2} \lim _{r \rightarrow \infty}\left(r^{2} \frac{d \Phi}{d r}\right)\right]=\frac{1}{\Phi_{\infty}}\left(M_{A D M}-\frac{1}{2} \widetilde{Q}_{S}\right)
$$

so called because it is the active gravitational mass which affects the motion of a test particle composed entirely of gravitational field self energy (such as a black hole). The second equality in eqn (37) defines the scalar charge $\widetilde{Q}_{S}$ associated with $\Phi$, which characterises the extent to which the gravitational energy of the star differs from that of a similar star in GR, and we use a tilde to denote quantities relating to BD theory. Equation (37) includes an overall factor of $\Phi_{\infty}^{-1}$ to account for the fact that, as $\Phi$ varies, the strength of the gravitational coupling $G$ varies and the gravitational energy, which includes implicitly a factor of $G$, should vary in the same way. 
To find an expression for the energy of a solution of the VSL theory, we shall find an expression for the Hamiltonian. The procedure is almost identical to that given for dilaton theories by Ho, Kim and Park [15, so we only give the result here. We consider only a static spacetime; the generalisation to a time-dependent spacetime is as straight forwards as it is in GR. We assume that the spacetime is asymptotically flat and use the usual $3+1$ splitting, denoting spacelike hypersurfaces by $\Sigma$ and the hypersurface orthogonal unit timelike vectors by $U^{a}$. The timelike, 2-dimensional boundary of integration at spacelike infinity we denote by $\mathcal{S}_{\infty}$ and its outward pointing, spacelike unit normal by $n^{a}$. Starting with the action (11), decomposing $\mathcal{R}$ into the Ricci scalar ${ }^{(3)} \mathcal{R}$ of the spacelike hypersurfaces and a divergence term, and integrating by parts several times, gives the VSL Hamiltonian

$$
\begin{array}{r}
H=-\int_{\Sigma} d^{3} x \sqrt{g}\left[\frac{e^{\epsilon \phi}}{16 \pi G}\left({ }^{(3)} \mathcal{R}-\left(\kappa+2 \epsilon^{2}\right) h^{i j} \partial_{i} \phi \partial_{j} \phi-2 \epsilon \Delta \phi\right)+e^{\beta \phi} L_{m}\right] \\
+\frac{1}{8 \pi G} \int_{\mathcal{S}_{\infty}} d^{2} x \sqrt{B \sigma} e^{\epsilon \phi}\left(\theta-\epsilon n^{a} \partial_{a} \phi\right),
\end{array}
$$

where $\theta$ is the extrinsic curvature scalar of, and $\sigma_{a b}$ the induced metric on, the surface $\mathcal{S}_{\infty}$. The integral over $\Sigma$ vanishes by virtue of Hamilton's equation $\delta H / \delta \sqrt{B}=0$, where $\sqrt{B}$ is the lapse function, and we are left with the surface term. In general, this diverges and we must subtract a reference term $H_{B}$. Following [16, we choose $H_{B}$ to have the form

$$
H_{B}=\frac{1}{8 \pi} \int_{\mathcal{S}_{\infty}} d^{2} x \sqrt{B \sigma_{B}} e^{\epsilon \phi_{B}} \theta_{B}
$$

where the subscript " $B$ " denotes quantities evaluated when $\Sigma$ is intrinsically flat. The Hamiltonian is then

$$
H=\frac{1}{8 \pi} \int_{\mathcal{S}_{\infty}} d^{2} x \sqrt{B \sigma} e^{\epsilon \phi}\left(\theta-\epsilon n^{a} \partial_{a} \phi\right)-H_{B}
$$

We assume that $\phi$ is constant over $\mathcal{S}_{\infty}$ and denote its value by $\phi_{\infty}$. We further assume the $\phi_{B}=\phi_{\infty}$. One can then show that eqn (40) reduces to

$$
H=e^{\epsilon \phi_{\infty}}\left(M_{A D M}-\frac{1}{2} \epsilon Q_{S}\right)
$$

where

$$
Q_{S}:=\frac{1}{4 \pi} \int_{\mathcal{S}_{\infty}} n^{a} \partial_{a} \phi
$$

is the scalar charge associated with $\phi$. In a spherically symmetric spacetime,

$$
Q_{S}=\lim _{r \rightarrow \infty}\left(r^{2} \frac{d \phi}{d r}\right)
$$

As one would expect from the form of the VSL action, eqn (41) is similar to eqn (37).

There is an ambiguity in the derivation of eqn (41) in that it is not immediately clear if one should multiply $H$ by an additional power of $e^{\phi_{\infty}}$ to account for the effect on the total energy of varying the value of $c$ throughout a solution. This is related to the problem discussed in [6] of deciding on the power of $c_{\infty}$ one must multiply the action (11) by. To show that in fact eqn (41) needs no such additional factor we consider the energy of a star in the weak field limit. Combining the second equality of eqn (27) with the definition of pressure $p:=m c^{2} n^{2} \partial \Pi / \partial n$, one can show that

$$
(\rho+p) n^{\prime}=n p^{\prime}
$$


Combining this with eqn (12) and integrating gives

$$
m c^{2} n=(\rho+p) \sqrt{B} e^{\beta \phi} .
$$

We can define a "rest energy" $M_{R}=m c^{2} N$ for the star, which is simply the sum of the energies of its constituent particles and is its total energy in the weak field limit. Combining eqns (30) and (45) we have

$$
M_{R}=\int_{0}^{\infty} 4 \pi r^{2} \sqrt{B} A^{3 / 2} e^{\beta \phi}(\rho+p) d r .
$$

In the lowest order weak field limit, $\rho>>p, g_{a b} \rightarrow \eta_{a b}$ and $\phi$ is constant. In this case, eqns (2) and (34) together imply that the weak field ADM mass is

$$
M_{A D M}=\int_{0}^{\infty} 4 \pi r^{2} \rho e^{(\beta-\epsilon) \phi_{\infty}} d r
$$

while the Hamiltonian in this limit is simply $e^{\epsilon \phi_{\infty}} M_{A D M}$. Under the lowest order weak field approximation, this is identical to the quantity defined by eqn (46) indicating that no additional powers of $e^{\phi_{\infty}}$ need be included in the energy definition and so we shall adopt eqn (41) as the definition of the energy of the star. Due to this expression's similarity with eqn (37), we shall henceforth denote the energy of VSL solutions by $M_{T}$.

We may check that our definitions of energy and particle number, defined by eqn (30) are consistent as follows. A necessary condition for the stability of stellar solutions in any theory of gravity is that, given a set of solutions parameterised by a smoothly varying quantity such as their central density, the extremal values of the total energy and the conserved particle number should coincide [17]. Using a method similar to the one outlined in [18 for boson-fermion stars, it is relatively easy to prove that $N$ defined by eqn (30) and our choice of eqn (41) for the energy of a solution are consistent with this requirement in that these two quantities do have coinciding extrema.

In a metric theory, $M_{P}$ defined by eqn (36) is also the Kepler mass $M_{K}$ of the star. However, this is not the case here: the Kepler mass in a VSL theory includes a term proportional to $\beta$ which embodies the non-minimal coupling between $L_{m}$ and the metric and, in terms of the variables we are using here, is given by [7]

$$
M_{K}=M_{P}+\beta Q_{S},
$$

where, in terms of $M_{A D M}$ and $Q_{S}$, the Poisson mass is

$$
M_{P}=M_{A D M}-\epsilon Q_{S}
$$

These definitions are similar to those in BD theory except that in the later case, $M_{P}$ and $M_{K}$ are identical.

\section{$6 \quad$ Numerical Results}

Equations (92) to (12) are invariant under the rescaling

$$
\phi \rightarrow \phi+s, \quad A \rightarrow A e^{(\epsilon-\beta) s} .
$$

We use this property to set $\phi_{\infty}=0$ for all solutions. In addition, eqns (91) to (12) are invariant under the rescaling

$$
A \rightarrow a^{2} A, \quad r \rightarrow \frac{r}{a}, \quad Z \rightarrow a Z
$$


and we use this to set $A_{\infty}=1$ for all solutions. Note that this last rescaling implies that $\mu \rightarrow \mu / a$. The function $Z$ automatically satisfies the boundary condition $Z_{\infty}=0$. We calculate $\mu, C$ and $\lambda$ for each solution using eqns (19) to (21), in which we evaluate $A, Z, \phi$ and their derivatives at the outermost point of the interior solution. Equation (16) is then used to check the consistency of the numerical calculations. We characterise the size of each solution by the Schwarzschild radius $R$, which is related to $r$ by

$$
R:=\sqrt{A_{s}} r_{s}
$$

where the subscript $s$ denotes quantities evaluated at the surface of the star. This is the same definition of radius used in [13.

Figure 1 shows curves of mass against central density for several sets of solutions with EOS 28. For each set, the value of $\epsilon$ is fixed, we impose the condition $\epsilon-\beta=4$ and choose the minimum value of $\kappa$ consistent with eqn (26). The mass of each solution is calculated using eqn (41). The $\epsilon=4$ solutions are indistinguishable from those in GR since they are identical to solutions in BD theory with a large value of $\omega$. The Figure shows that as $\epsilon$ and $\kappa$ decrease, the energy of all of the solutions decreases. Masses $M_{T}$ and $M_{K}$, along with other data from the same sets of solutions, are shown in Table 1] we do not show values of $M_{P}$ since they are very close to corresponding values of $M_{T}$. The data in the table indicate that, as well as the mass, the radii of the solutions also decreases rapidly with $\epsilon$.

\begin{tabular}{|c|c|c|c|c|c|c|}
\hline$\epsilon$ & $\kappa$ & $\kappa / \epsilon^{2}$ & $M_{T}$ & $M_{K}$ & $Q_{S}$ & $R$ \\
\hline 4.0 & 53290 & 3330 & 0.711 & 0.712 & -0.0000 & 10.1 \\
1.0 & 13330 & 13330 & 0.699 & 0.712 & -0.0036 & 10.0 \\
0.1 & 1333 & $1.33 \times 10^{5}$ & 0.588 & 0.711 & -0.0311 & 9.50 \\
0.005 & 666 & $2.67 \times 10^{5}$ & 0.495 & 0.704 & -0.0524 & 8.99 \\
0.001 & 13.3 & $1.33 \times 10^{7}$ & 0.192 & 0.598 & -0.1017 & 6.62 \\
0.0005 & 6.66 & $2.67 \times 10^{7}$ & 0.096 & 0.502 & -0.1016 & 5.27 \\
0.0001 & 1.33 & $1.33 \times 10^{8}$ & 0.012 & 0.269 & -0.0642 & 2.66 \\
\hline
\end{tabular}

Table 1: VSL neutron stars with $\epsilon-\beta=4$ and central density $\rho_{0}=2.9 \times 10^{18} \mathrm{~kg} \mathrm{~m}^{-3}$. The energy $M_{T}$ is calculated using eqn (41), the Kepler mass $M_{K}$ using eqn (48) and $Q_{S}$ is the scalar charge associated with $\phi$. All three are measured in units of solar mass. The radius, in units of $\mathrm{km}$, is calculated using eqn (52).

The response of the properties of the VSL stars to changes in the scalar field coupling strength is in marked contrast to to that for neutron stars in BD theories, data for which are shown in Table 2. (Further data on the structure of BD stars can be found in Salmona 13] but note that the mass used in this reference is the Poisson mass and the boundary value of $\Phi$ is different from our value of $\Phi_{\infty}=1$.) Values of $\omega$ have been chosen in the Table to match those of $\kappa$ in Table 1 and we show masses $M_{T}$ and $M_{K}$; the Poisson mass $M_{P}$ is identical to $M_{K}$. The data show that increasing the strength of the scalar field's coupling has a moderate effect in decreasing the energy and radius of the stars, while increasing the Poisson mass.

There are two reasons for the differences between BD and VSL neutron stars' properties. From eqn (11), for small values of $\epsilon$, the strength of the coupling between $\phi$ and the matter varies as $(\kappa / \epsilon)^{-1}$. This quantity increases as $\kappa$ decreases, which means that the $\phi$ field becomes more inhomogeneous and the magnitude of $Q_{S}$ increases as we decrease $\epsilon$. This behaviour is very similar to that for BD theory. However, in contrast with BD theory, the ADM mass, Poisson mass and energy $M_{T}$ differ by multiples of $\epsilon Q_{S}$ and the product of these two quantities 


\begin{tabular}{|c|c|c|c|c|}
\hline$\omega$ & $M_{T}$ & $M_{P}$ & $\widetilde{Q}_{S}$ & $R$ \\
\hline 1333 & 0.711 & 0.711 & -0.0004 & 10.1 \\
666 & 0.711 & 0.712 & -0.0008 & 10.1 \\
13.3 & 0.705 & 0.723 & -0.0365 & 10.1 \\
6.66 & 0.699 & 0.732 & -0.0655 & 10.0 \\
1.33 & 0.673 & 0.764 & -0.1818 & 9.95 \\
\hline
\end{tabular}

Table 2: Neutron stars on BD theory with central density $\rho_{0}=2.9 \times 10^{18} \mathrm{~kg} \mathrm{~m}^{-3}$. The energy $M_{T}$ is calculated using eqn (37), the Poisson mass $M_{P}$ using eqn (36) and $\widetilde{Q}_{S}$ is the scalar charge associated with the BD scalar field $\Phi$. All three are measured in units of solar mass. The radius, in units of $\mathrm{km}$, is calculated using eqn (52). For BD theory, the Poisson and Kepler masses are identical.

decreases as $\epsilon$ decreases. This is in contrast with BD theory, where the larger value of $\widetilde{Q}_{S}$ serves to increase the Poisson (or Kepler) mass to a value significantly larger that other masses.

The second, and more significant, cause of structure difference between the two theories is due to the non-universal coupling between the matter and the metric in the VSL action. From eqn (12), the pressure gradient has a term involving the product $\beta \phi^{\prime}$. For all of these solutions, $\phi^{\prime}<0$ and, under the assumption that $\epsilon-\beta=4, \beta<0$. Hence the product $\beta \phi^{\prime}>0$ and since $p$ must be a decreasing function of $r$, eqn (12) implies that the term involving $\beta$ increases the magnitude of the pressure gradient, leading to $p \rightarrow 0$ at a smaller value of $R$ than for a star in metric theory with an identical central density. The significantly smaller physical size of the star means that its energy is also much smaller.

Figure 2 shows mass against central density for sets of solutions with EOS 29] with the same limits on the parameters $\epsilon$ and $\beta$ as for the solutions of Figure 1. These solutions show the same overall behaviour as those with the simpler EOS: as the parameter $\eta$ decreases, the solutions become less massive for a given central density. As before, the $\epsilon=4$ solutions are indistinguishable from those of GR. In addition, the central density at which the maximal mass solution occurs increases as $\epsilon$ decreases, again in a similar way to the solution shown in Figure 1. In addition we have found that, for a given central density, the radius of a solution decreases as $\epsilon$ decreases. However, both this feature and the reduction in mass are less severe than for the simpler EOS and both maximal mass and minimum radius of the maximal mass solution decrease far less rapidly with decreasing $\epsilon$. This is due to the difference in the stiffness between the two equations of state.

Since the Figure 2 solutions have a reasonably realistic EOS, we can use them to place a limit on the parameters of the VSL theory for the case studied here in which $\epsilon-\beta=4$. The masses of neutron stars in binary systems detected to date have masses in the range (1.36 \pm 0.8$)$ solar masses, while neutron stars in orbit about other types of companion body have masses of at least 1.4 solar masses (for a review of this data and references, see 21]). Hence, for a VSL theory to be viable, it must allow for the existence of neutron stars whose mass is at least 1.3 solar masses. From Figure 2, this implies that the parameter $\epsilon$ must satisfy the inequality $\epsilon \geq 0.00015$. 


\section{Sensitivity and Evolution of Mass}

For many theories of gravity, such as BD theory and other more general scalar-tensor (ST) theories, solar system observations place strong constraints on the theories' parameters via the PPN formalism, while limits derived from observation of strong field phenomena are far weaker. However, for VSL theories with small values of $\epsilon$, the PPN limit (26) on $\kappa$ is extremely weak and we should therefore look to strong field observations, in particular binary pulsar data, to provide the strongest constraints. The situation here is similar to that found by Damour and Esposito-Farese for certain classes of ST theory which satisfy PPN limits to an arbitrarily high degree but show significant strong field effects [10] and references therein. We shall leave the full treatment of the motion of binary pulsars in a VSL theory to a future publication. Here we shall briefly mention some of the key ideas involved and use part of the formalism to discuss how VSL stars will evolve in a cosmological setting.

The analysis of binary pulsar motion is based on the parameterised post-Keplerian (PK) formalism, which treats each star as a point mass moving according to quasi-Newtonian equations of motion. Details of the particular theory under study are made manifest by allowing the active, passive and inertial masses of each star to vary according to the value of any ambient, non-metric gravitational fields. At the lowest level of approximation, the variability of a particular mass is quantified by the sensitivity $s$, given by

$$
s:=\left.\sum_{A} \frac{\partial(\log M)}{\partial\left(\log \psi^{A}\right)}\right|_{\psi_{\infty}^{A}},
$$

where $M$ is the mass under consideration and the $\psi^{A}$ are the non-metric gravitational fields. In ST theories of gravity, there is only one extra field, the scalar field $\Phi$ and it turns out that active, passive and inertial masses are proportional to each other. For the VSL theory we are considering here, we expect much of the formalism to be very similar to that for BD gravity, since the vacuum, inter-body field equations are the same for both theories. Since the BD and VSL scalar fields are related by $\Phi=e^{\epsilon \phi}$, we shall define $s$ to be

$$
s=\frac{\partial(\log M)}{\partial(\epsilon \phi)}=\frac{1}{\epsilon M} \frac{\partial M}{\partial \phi}
$$

where again $M$ denotes any mass.

We shall next derive an expression for the sensitivity associated with the Hamiltonian, identical to the VSL tensor mass $M_{T}$, of a compact object in a VSL theory. We consider first the variation of the Hamiltonian (38) induced by a variation of $\phi$ on $\mathcal{S}_{\infty}$. Taking the functional derivative of this equation, performing several integration by parts and noting that the volume term vanishes and the terms involving $\partial_{a}(\delta \phi)$ in the surface integral cancel, we are left with

$$
\delta H=\frac{1}{8 \pi G} \int_{\mathcal{S}_{\infty}} d^{2} x \sqrt{B \sigma} e^{\epsilon \phi}\left(\epsilon \theta-\epsilon n_{a} U^{b} \nabla_{b} U^{a}+\kappa n^{a} \partial_{a} \phi\right) \delta \phi_{\infty}+\delta H_{B},
$$

where we have assumed that $\delta \phi$ is constant over $\mathcal{S}_{\infty}$ and have denoted it by $\delta \phi_{\infty}$. The reference term $H_{B}$ is linear in $e^{\phi_{\infty}}$. Thus

$$
\delta H_{B}=\epsilon H_{B} \delta \phi_{\infty}
$$

Combining the above two equations with eqn (38), we have

$$
\delta H=\frac{1}{8 \pi G} \int_{\mathcal{S}_{\infty}} d^{2} x \sqrt{B \sigma} e^{\epsilon \phi}\left[\left(\kappa+\epsilon^{2}\right) n^{a} \partial_{a} \phi-\epsilon n_{a} U^{b} \nabla_{b} U^{a}\right] \delta \phi_{\infty}+\epsilon H \delta \phi_{\infty} .
$$


Using the definition (36), one can show that

$$
\frac{1}{8 \pi G} \int_{\mathcal{S}_{\infty}} d^{2} x \sqrt{B \sigma} n_{a} U^{b} \nabla_{b} U^{a}=\frac{1}{2} M_{P}
$$

In addition, the first term of eqn (57) is simply $\frac{1}{2}\left(\kappa+\epsilon^{2}\right) Q_{S}$. Thus we have

$$
\delta H=e^{\epsilon \phi_{\infty}}\left[\frac{1}{2}\left(\kappa+\epsilon^{2}\right) Q_{S}-\frac{1}{2} \epsilon M_{P}\right] \delta \phi_{\infty}+\epsilon H \delta \phi_{\infty} .
$$

Finally, using the relations (41) and (49), and taking the limit $\delta \phi_{\infty} \rightarrow 0$, one can show that

$$
\frac{\partial H}{\partial \phi_{\infty}}=\frac{1}{2} \epsilon H+e^{\epsilon \phi_{\infty}}\left(\frac{3 \epsilon^{2}+2 \kappa}{4}\right) Q_{S} .
$$

Hence the sensitivity associated with both $H$ and the identical quantity $M_{T}$ is

$$
s=\frac{1}{2}+e^{\epsilon \phi_{\infty}}\left(\frac{3 \epsilon^{2}+2 \kappa}{4 \epsilon}\right) \frac{Q_{S}}{M_{T}} .
$$

Due to the similarities between the two theories, we expect $s$ to appear in the PK formalism for a VSL theory in much the same way as in a ST theory. Thus we expect that, for any particular mass $M$, presence of the $\phi$ field introduces a strong field correction of the form $M \rightarrow M(1+\nu s)$, where $\nu$ is a parameter which depends the type of mass considered. Equation (61) then indicates how strong field gravitational effects depend upon the values of $\epsilon$ and $\beta$.

Equation (61) may be used to investigate the gravitational evolution of a compact body in a VSL theory. The scalar field far from a compact body such as a neutron star should match smoothly to the cosmological value of the field. Hence, assuming that a body embedded in a cosmological solution can be modelled approximately by an isolated, asymptotically flat solution, one expects that the boundary field $\phi_{\infty}$ to follow the cosmological evolution of $\phi$. Equation (61) then implies that the evolution of the mass of the body depends strongly on the relative values of $\epsilon$ and $\kappa$. For all of the solutions shown in Figure 1 and Table 1 except the $\epsilon=4$ solutions, $s<0$. The primary reason for this is that, as $\epsilon$ decreases, the ratio $\kappa / \epsilon$ which dominates the second term of eqn (61) increases dramatically. In a cosmological solution, $\phi$ should be a decreasing function of cosmological time so as to be consistent with the requirement that $c$ was larger in the past. Use of eqn (61) then implies that the energy of these VSL stars increases with cosmological time, the largest rates of increase coinciding with smallest values of $\epsilon$. This is in marked contrast with ST theories for which, in general, the energy of a compact body decreases with cosmological time [19, 20].

\section{Conclusions}

We have examined the properties of neutron stars in one particular VSL theory in which the local value of the speed of light is determined by a scalar field $\phi$. The theory bears a more than superficial resemblance to $\mathrm{BD}$ and other ST theories, and we have found that the expression giving the energy of an asymptotically flat solution to the VSL field equations is very similar to it counterpart in ST theories. However, despite these similarities, the properties of VSL neutron stars are very different to their counterparts in VSL theories. In particular, for certain choices of $\epsilon$ and $\beta$, VSL neutron stars are limited to being much smaller those in GR and the dependence of mass on the strength of the coupling between $\phi$ and the matter is extremely 
strong. This allows one to use the mere existence of neutron stars to place constraints on the theory's parameters, even without having to analyse the motion of binary pulsars: the maximum neutron star mass allowed by the theory must obviously be at least as large as those of observed neutron stars. Unfortunately, neutron star masses are fairly sensitive to the EOS used (see, for example, [11, 22]), although we have placed a rather crude limit on the parameter $\epsilon$ using a reasonably realistic equation of state.

We have also given a brief analysis of the evolution of a VSL star in a cosmological setting found that, as the cosmological background evolves and $\phi$ decreases in value, the energy of a VSL star increases in general. This result is independent of the EOS used or even of the type of matter of which the star is composed and could have serious implications for the stability of a star since its fractional binding energy $\left(M_{T}-N\right) / N$ could eventually become positive.

To properly place limits on the parameters of a VSL theory, one needs a full analysis of the predicted behaviour of binary pulsar motion. However, this is not the only area of astrophysics in which a VSL theory might give significantly different predictions to those of other theories. For example, it was shown in [7] that black holes in this theory are very different to their GR counterparts in that the proper time taken for an in-falling particle to reach the horizon can be infinite. If this is indeed the case, a varying speed of light may have a significant effect on the physics of black hole accretion disks since matter orbiting the black hole would never enter the black hole horizon.

\section{Acknowledgements}

I would like to thank the University of Sussex for its hospitality while this work was completed.

\section{References}

[1] J. D. Barrow and J. Magueijo, Physics Letters B, 443, 104 (1998).

[2] J. K. Webb, M. T. Murphy, V. V. Flambaum and S. J. Curran, Astrophysical Journal Supplement 203, 577 (2003).

[3] C. J. A. P. Martins, A. Melchiorro, G. Rocha, R. Trotta, P. P. Avelino and P.Viana, astro-ph/0302295.

[4] P. P. Avelino and C. J. A. P. Martins, Physical Review D 67, 027302 (2003).

[5] M. A. Clayton and J. W. Moffat, Physics Letters B506, 177 (2001).

[6] J Magueijo, Physical Review D 62, 103521 (2000).

[7] J Magueijo, Physical Review D 63, 043502 (2001).

[8] T. M. Eubanks et al, Bull. Am. Phys. Soc. Abstract K11.05 (1997).

[9] L. D. Landau and E. M. Lifshitz, The Classical Theory of Fields, Pergamon Press (1989).

[10] T. Damour and G. Esposito-Farese, Physical Review Letters, 70, 2220 (1993).

[11] J. Diaz-Alonso and J. M. Ibanez-Cabanell, Astrophysical Journal 291, 308 (1985).

[12] J. R. Oppenheimer and G. M. Volkoff, Physical Review 55, 374 (1938).

[13] A. Salmona, Physical Review 154, 1218 (1967). 
[14] D. L. Lee, Physical Review D 10, 2374 (1974).

[15] , J. Ho, Y. Kim and Y. J. Park, Modern Physics Letters A 11, 2037 (1996).

[16] J. D. Brown and J. W. York, Physical Review D, 47, 1407 (1993)

[17] F. V. Kusmartsev, E. W. Mielke and F. Schunck, Physical Review D 43, 3895 (1991).

[18] P. Jetzer, Physics Letters B, 231, 433 (1989).

[19] A. W. Whinnett, Physical Review D, 61, 124014 (2000).

[20] G. L. Comer and H. Shinkai, Classical \& Quantum Gravity, 15, 669 (1998).

[21] Introductory talk given at the Compact Stars in the QCD Phase Diagram conference, Copenhagen, 2001, astro-ph 0201465.

[22] W. D. Arnett and R. L. Bowers, Astrophysical Journal Supplement 33, 415 (1977). 


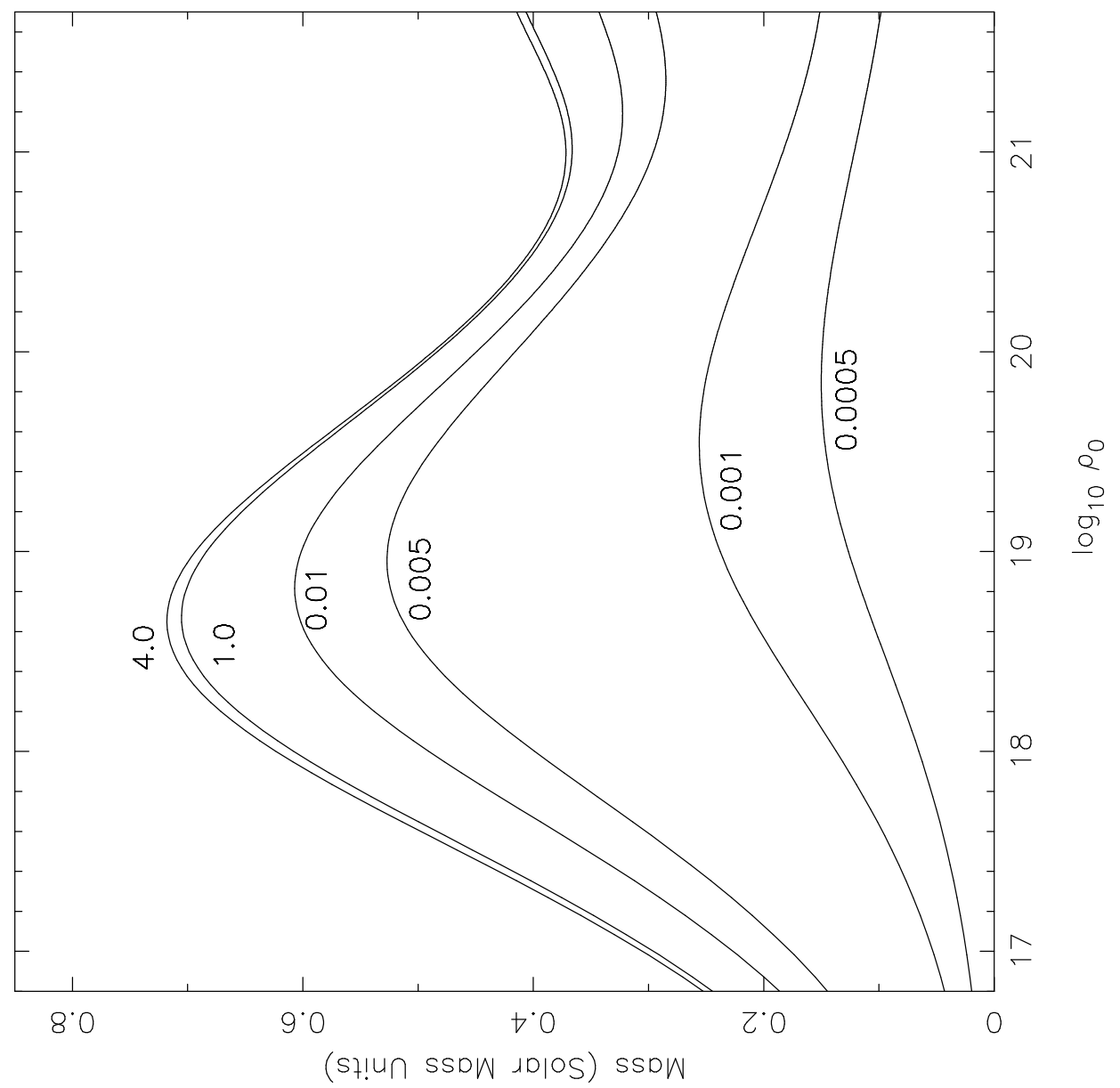

Figure 1: Mass curves for neutron stars in the VSL theory with the simple, non-interacting Fermion EOS (28). The curves are labelled by their value of $\epsilon$. For each curve, $\epsilon-\beta=4$ and the value of $\kappa$ is the minimum value consistent with the weak field, solar system constraints. The central density is given in units of $\mathrm{kg} \mathrm{m}^{-3}$. 


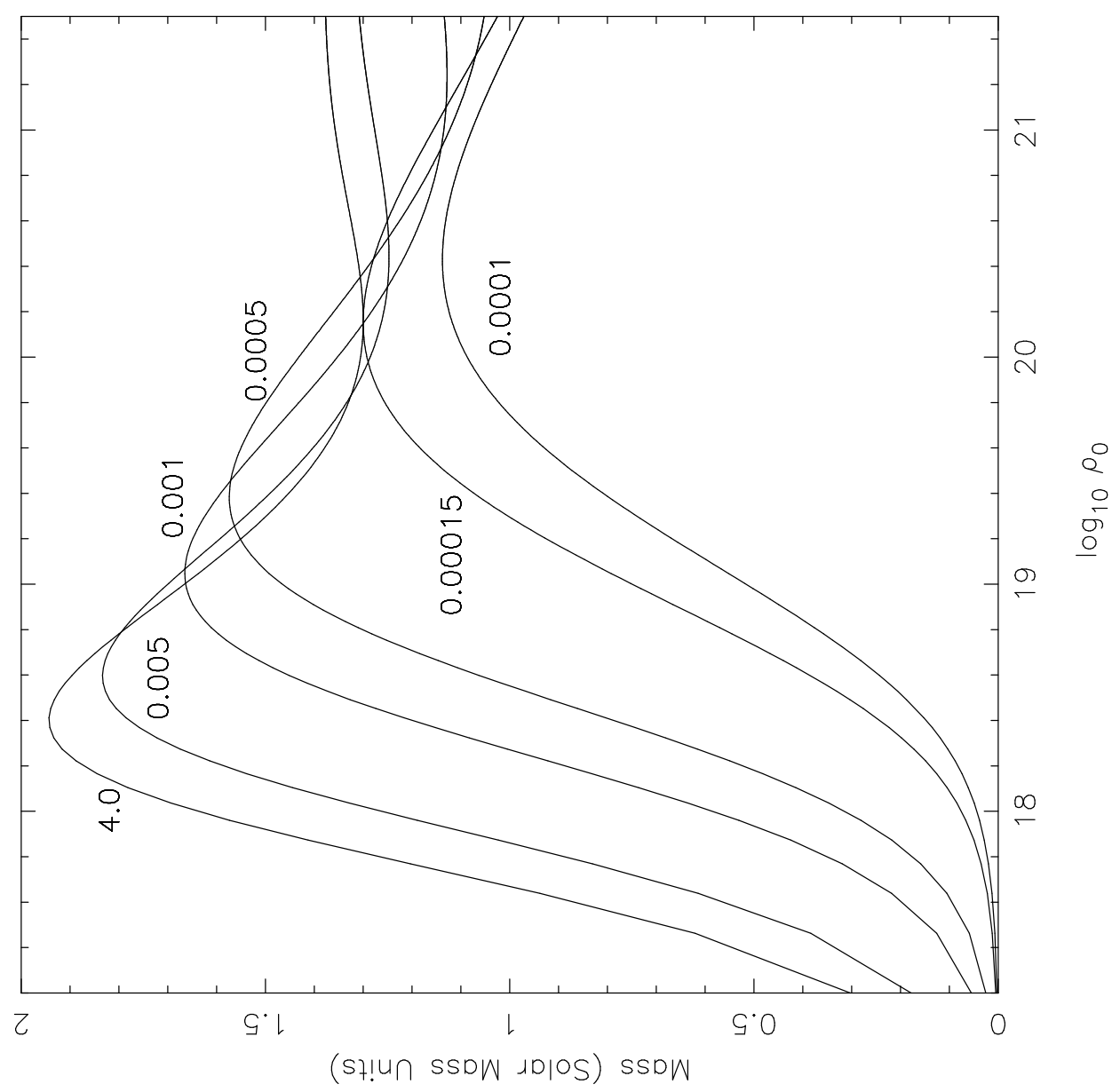

Figure 2: Mass curves for neutron stars in the VSL theory with the realistic EOS (29). Units, labels and curve parameterisation is the same as in Figure 1. 